PESQUIMAT 24(2): 45-59(2021)

ISSN:1560-912X/ ISSN-E:1609-8439

https://doi.org/10.15381/pesquimat.v24i2.21523

Facultad de Ciencias Matemáticas - UNMSM

\title{
Comportamiento asintótico y simulación numérica por diferencia finita para el modelo de vibración de un sólido lineal estándar con efecto térmico
}

\author{
Carlos Javier Romero Figueroa ${ }^{1}$, Andrés Guardia Cay $d^{2}$ y Alfonso Pérez Salvatierra ${ }^{3}$
}

Resumen: En este artículo aplicando la teoría de semigrupo de operadores lineales, se estudia el decaimiento exponencial de las soluciones de un sistema acoplado que modela las vibraciones de un sólido lineal estándar con un efecto térmico disipativo. Con el fin de verificar numéricamente los resultados analíticos establecidos, se realiza una serie de simulaciones numéricas en su forma unidimensinal, para el cual consideramos el esquema numérico aplicando diferencias finitas.

Palabras clave: Energía, semigrupo, estabilidad exponencial, simulación numérica.

\section{Asymptotic behavior and numerical simulation by finite difference for the vibration model of a standard linear solid with thermal effect}

\begin{abstract}
In this article applying the semigroup theory of linear operators, we study the asymptotic behavior of the solutions of a coupled system that models the vibrations of a standard linear solid with a dissipative thermal effect. In order to verify numerically the established analytical results, a series of numerical simulations are carried out in their one-dimensional form, for which we consider the numerical scheme applying finite differences.
\end{abstract}

Keywords: Energy, semigroup, exponential stability, numerical simulation.

Recibido: 15/11/2021. Aceptado: 03/12/2021. Publicado online: 30/12/2021.

(C) Los autores. Este artículo es publicado por la Revista PESQUIMAT de la Facultad de Ciencias Matemáticas, Universidad Nacional Mayor de San Marcos. Este es un artículo de acceso abierto, distribuido bajo los términos de la licencia Creative Commons Atribucion-No Comercia-CompartirIgual 4.0 Internacional.(http://creativecommons.org/licenses/by-nc-sa/4.0/) que permite el uso no comercial, distribución y reproducción en cualquier medio, siempre que la obra original sea debidamente citada. Para uso comercial, por favor póngase en contacto y reproduccion en cualquier medio, siempre que

${ }^{1}$ UNMSM, Facultad de Ciencias Matemáticas. e-mail: carlos.romero1@unmsm.edu.pe

${ }^{2}$ UNMSM, Facultad de Ciencias Matemáticas, e-mail: aguardiac@unmsm.edu.pe

${ }^{3}$ UNMSM, Facultad de Ciencias Matemáticas, e-mail: aperezs@unmsm.edu.pe 


\section{Introducción}

En el presente artículo aplicando la técnica de semigrupos vamos a establecer la estabilidad exponencial del $C_{0}$-semigrupo asociado a un sistema acoplado que modela las vibraciones de un sólido lineal estándar con efecto térmico disipativo. Finalmente, llevaremos acabo un análisis cualitativo del sistema en estudio, en su forma unidimensional vía el método de diferencia finitas con el fin de verificar numéricamente los resultados que se irán estableciendo en el desarrollo del presente trabajo. Las ecuaciones que modelan el fenómeno son

$$
\begin{aligned}
\alpha u_{t t t}+u_{t t}-a^{2} \Delta u-a^{2} \beta \Delta u_{t}+\eta \Delta \theta & =0, \text { en } \Omega \times] 0, \infty[ \\
\theta_{t}-\Delta \theta-\alpha \eta \Delta u_{t t}-\eta \Delta u_{t} & =0, \text { en } \Omega \times] 0, \infty[
\end{aligned}
$$

Condiciones iniciales

$$
u(x, 0)=u_{0}(x), u_{t}(x, 0)=u_{1}(x), u_{t t}(x, 0)=u_{2}(x), \theta(x, 0)=\theta_{0}(x), \text { en } \Omega
$$

Condiciones de frontera

$$
u=0, u_{t}=0, \theta=0, \text { sobre } \partial \Omega=\Gamma
$$

Donde $\Omega$, es un subconjunto abierto, acotado de $\mathbb{R}^{n}$ para $n \geq 1$, con frontera $\Gamma=\partial \Omega$ suficientemente regular.

En el sistema acoplado (1)-(4), la función $u=u(x, t)$ representa la vibración de la estructura flexible y $\theta=\theta(x, t)$ representa la diferencia de temperatura entre la temperatura actual y la de referencia. Las constantes $\alpha, a, \beta$ y $\eta$ son positivas, siendo $\eta$ la constante de acoplamiento.

Es de mencionar que la mayoría de los sólidos tienen una estructura periódica de átomos, que forman lo que llamamos una red cristalina. Los sólidos y cristales amorfos son excepciones. La existencia de la red cristalina, implica un grado de simetría en la disposición de la red. Estas simetrías existentes se han estudiado ampliamente. La estructura cristalina es una de las características de los minerales. Una de las implicaciones de la red simétrica de átomos, es que puede soportar modos de vibración de red resonantes. Estas vibraciones transportan energía y son importantes en la conductividad térmica de los elementos no metálicos, y en la capacidad calorífica de todos los sólidos.

De hecho, estudios recientes sobre estabilización de sistemas mecánicos han ganado importancia debido a la aplicación del control de vibración en varios elementos estructurales. La dinámica de las vibraciones lineales de la estructura elástica está matemáticamente gobernada por la ecuación de onda,

$$
y^{\prime \prime}(x, t)=\sigma^{2} \Delta y(x, t)
$$

en algún dominio $\Omega$ y $\sigma>0$ es la velocidad de onda constante.

La dinámica de la ecuación (5) se formula sobre la base de la ley de Hook, en la cual la tension $\sigma$ es simplemente proporcional a la deformación $e$, lo que significa que $\sigma=E e, E$ es el módulo de Young de la estructura elástica, sin embargo, la dinámica de las vibraciones elásticas de las estructuras flexibles en la práctica no es lineal. En realidad es bastante engorroso para un tratamiento analítico del problema, cabe precisar ocurren por lo general, cuando es no lineal y, por lo tanto el resultado así obtenido generalmente no será de forma precisa.

En el artículo Gorain [5], busca un modelo más realista de vibraciones no lineales de estructura elástica comúnmente conocido como el modelo lineal estándar "de viscoelasticidad (cf. Fung [7]), en el que la tensión $\sigma$ no es simplemente proporcional a la tensión $e$. En este modelo, un resorte lineal está conectado en serie con una combinación de otro resorte lineal y un tablero en paralelo y la fórmula correspondiente de tensión-deformación de la estructura elástica se describe por la relación constitutiva; estudiado por Fung [7] y Rabotnov [13]

$$
\sigma+\lambda \sigma^{\prime}=E\left(e+\mu e^{\prime}\right)
$$


Donde $\lambda, \mu$ son constantes pequeñas que satisfacen la relación $0<\lambda<\mu$.

Como resultado de esto, la dinámica de las vibraciones de las estructuras elásticas se rige con mayor precisión por la ecuación diferencial de tercer orden,

$$
\lambda y^{\prime \prime \prime}(x, t)+y^{\prime \prime}(x, t)=c^{2}\left(\Delta y(x, t)+\mu \Delta y^{\prime}(x, t)\right)
$$

El autor estudia la estabilización del problema matemático $(7)$ en un dominio $\Omega \times \mathbb{R}^{+}$, bajo la condiciones de frontera mixtos no amortiguadas

$$
y=0 \text { sobre } \Gamma_{0} \times \mathbb{R}^{+}, \frac{\partial y}{\partial \nu}=0 \text { sobre } \Gamma_{1} \times \mathbb{R}^{+}
$$

Bajo las condiciones iniciales

$$
y(x, 0)=y_{0}(x), y^{\prime}(x, 0)=y_{1}(x) \text { y } y^{\prime \prime}(x, 0)=y_{2}(x) \text { en } \Omega
$$

donde $\Omega$ es un conjunto conexo limitado en $\mathbb{R}^{n}$, para $n \geq 1$, con frontera regular $\Gamma=\partial \Omega$ que consta de dos partes $\Gamma_{1}$ y $\Gamma_{0}$ de modo que $\Gamma_{1} \cup \Gamma_{2}=\Omega$ y $\overline{\Gamma_{1}} \cap \overline{\Gamma_{0}}=\emptyset$, aquí $\nu$ denota la unidad normal de $\Gamma$ apuntando hacia el exterior de $\Omega$ y $\left.\mathbb{R}^{+}=\right] 0 ;+\infty[$.

\section{Preliminares}

Enunciamos algunos resultados de la teoría de semigrupos lineales, que serán utilizados en el presente trabajo.

Teorema 1 (Prüss) Sea $A$ el generador infinitesimal de un semigrupo de contracciones $\{T(t)\}_{t \geq 0}$, entonces el semigrupo $T(t)$ es exponencialmente estable si y solo si

a) $i \mathbb{R} \subset \rho(A)$

b) $\lim _{|\lambda| \rightarrow \infty} \sup \left\|(i \lambda I-A)^{-1}\right\|_{L(\mathcal{H})}<\infty$

Demostración. Ver [12.

Teorema 2 Sea $A$ un operador lineal y cerrado en un espacio de Hilbert $H$ tal que el operador resolvente $\left(\lambda_{0} I-A\right)^{-1}$ existe y es compacto para algún $\lambda_{0}$. Entonces el espectro $\sigma(A)=\mathbb{C} \backslash \rho(A)$ está conformado únicamente por autovalores de $A$ con multiplicidad finita.

Demostración. Ver Kato T. 8], página 187.

\section{Existencia y unicidad de soluciones}

En esta sección, establecemos la existencia y unicidad de soluciones del sistema (1)-(2), con condiciones iniciales (3) y de frontera (4) a través de la teoría de semigrupos lineales, ver, por ejemplo Liu and Zheng [10]. y Pazy [11].

La teoría de semigrupos se desarrolla a partir de ecuaciones de primer orden en el tiempo. Para esto es necesario convertir el modelo anterior a un sistema de primer orden. Para tal consideremos la siguiente notación vectorial $U=(u, v, w, \theta)$ con $v=u_{t}, w=v_{t}$.

Entonces el problema (1)-(2) con condiciones iniciales y de frontera (3) y (4) se puede reescribir como el siguiente problema de valor inicial abstracto para una ecuación de evolución de primer orden

$$
U_{t}=A U, \quad U(0)=U_{0}
$$


donde $U_{0}=\left(u_{0}, v_{0}, w_{0}, \theta_{0}\right)^{T}$ y $A$ es el operador diferencial dado por

$$
A=\left(\begin{array}{cccc}
0 & I & 0 & 0 \\
0 & 0 & I & 0 \\
\frac{a^{2}}{\alpha} \Delta & \frac{a^{2} \beta}{\alpha} \Delta & -\frac{1}{\alpha} I & -\frac{\eta}{\alpha} \Delta \\
0 & \eta \Delta & \alpha \eta \Delta & \Delta
\end{array}\right)
$$

Teniendo en cuenta la condición de frontera (4), consideramos el espacio de Hilbert

$$
\mathcal{H}=H_{0}^{1}(\Omega) \times H_{0}^{1}(\Omega) \times L^{2}(\Omega) \times L^{2}(\Omega)
$$

Proposición 1 Si $(\beta-\alpha)>0$, entonces la energía del sistema (1)-(4) $E(t): \mathbb{R}^{+} \longrightarrow \mathbb{R}^{+}$dada en el tiempo $t$ está definida como

$$
E(t)=\frac{1}{2} \int_{\Omega}\left(\left|\alpha u_{t t}+u_{t}\right|^{2} d x+a^{2}\left|\alpha \nabla u_{t}+\nabla u\right|^{2}+a^{2} \alpha(\beta-\alpha)\left|\nabla u_{t}\right|^{2}+|\theta|^{2}\right) d x
$$

y satisface la ley de disipación

$$
\frac{d}{d t} E(t)=-a^{2}(\beta-\alpha) \int_{\Omega}\left|\nabla u_{t}\right|^{2} d x-\int_{\Omega}|\nabla \theta|^{2} d x
$$

Demostración. En efecto, realizando el cambio $\varphi=\alpha u_{t}+u$, en el sistema (1)-(4) y efectuando opeaciones pertinentes se obtiene la Proposición 1 .

De este modo se define el espacio de fase

$$
\mathcal{H}=H_{0}^{1}(\Omega) \times H_{0}^{1}(\Omega) \times L^{2}(\Omega) \times L^{2}(\Omega) .
$$

el cuál equipado con el siguiente producto interno

$$
\begin{aligned}
\left\langle U_{1}, U_{2}\right\rangle_{\mathcal{H}}=a^{2} \alpha(\beta-\alpha) \int_{\Omega} \nabla v_{1} \cdot \nabla \bar{v}_{2} d x & +a^{2} \int_{\Omega}\left(\nabla u_{1}+\alpha \nabla v_{1}\right)\left(\nabla \bar{u}_{2}+\alpha \nabla \bar{v}_{2}\right) d x \\
& +\int_{\Omega}\left(v_{1}+\alpha w_{1}\right)\left(\bar{v}_{2}+\alpha \bar{w}_{2}\right) d x+\int_{\Omega} \theta_{1} \bar{\theta}_{2} d x
\end{aligned}
$$

donde $U_{1}=\left(u_{1}, v_{1}, w_{1}, \theta_{1}\right), U_{2}=\left(u_{2}, v_{2}, w_{2}, \theta_{2}\right)$, es un espacio de Hilbert, con la norma dada por

$$
\|U\|_{\mathcal{H}}^{2}=a^{2} \alpha(\beta-\alpha)\|\nabla v\|_{L^{2}(\Omega)}^{2}+a^{2}\|\nabla u+\alpha \nabla v\|_{L^{2}(\Omega)}^{2}+\|v+\alpha w\|_{L^{2}(\Omega)}^{2}+\|\theta\|_{L^{2}(\Omega)}^{2}
$$

esta norma es equivalente a la norma usual

$$
\|U\|_{\widehat{\mathcal{H}}}^{2}=\|u\|_{H_{0}^{1}(\Omega)}^{2}+\|v\|_{H_{0}^{1}(\Omega)}^{2}+\|w\|_{L^{2}(\Omega)}^{2}+\|\theta\|_{L^{2}(\Omega)}^{2}
$$

Habiéndose definido el espacio de fase, se puede definir el dominio del operador $A$ de la siguiente forma. Recordando que en términos generales

$$
D(A)=\{U \in \mathcal{H} ; \quad A U \in \mathcal{H}\}
$$

Usando la definición del operador $A$ se obtiene

$$
\begin{gathered}
D(A)=\left\{(u, v, w, \theta) \in \mathcal{H}: w, \theta \in H_{0}^{1}(\Omega), a^{2} u+a^{2} \beta v-\eta \theta \in H_{0}^{1}(\Omega) \cap H^{2}(\Omega),\right. \\
\left.\eta v+\alpha \eta w+\theta \in H_{0}^{1}(\Omega) \cap H^{2}(\Omega)\right\}
\end{gathered}
$$

En consecuencia, el resultado de existencia y unicidad del sistema (1)-(2) con condiciones iniciales y de frontera (3) y (4) se dará a través del problema equivalente (8).

En estas condiciones, tenemos 
Teorema 3 Supongamos que $U_{0}=\left(u_{0}, v_{0}, w_{0}, \theta_{0}\right) \in D(A)$. Entonces el problema (8) posee una única soución $U=U=(u, v, w, \theta)$ en la clase "

$$
U \in C\left(\left[0, \infty[; D(A)) \cap C^{1}([0, \infty[; \mathcal{H}) .\right.\right.
$$

Demostración. No es difícil probar que $A$ es un operador disipativo en $\mathcal{H}, D(A)$ está densamente definido en $\mathcal{H}$, y $0 \in \rho(A)$, donde $\rho(A)$ es el conjunto resolvente de $A$. Por lo tanto, del Teorema de Lummer-Phillips, ver, por ejemplo Pazy [11]; Teorema 4.3, el operador $A$ es el generador infinitesimal de un $C_{0}$-semigrupo de contracciones $S(t)=e^{A t}$ sobre $\mathcal{H}$. Con lo que finaliza la demostración del teorema.

\section{Decaimiento exponencial}

En esta sección vamos a establecer el decaimiento exponencial de las soluciones del sistema acoplado que modela las vibraciones de un sólido lineal estándar con efecto térmico disipativo dada por (1)-(4), para el cual vamos a utilizar los resultados obtenidos por Prüss [12].

En el siguiente teorema, enunciamos el resultado central de esta sección.

Teorema $4 \mathrm{Si}(\beta-\alpha)>0$, entonces el semigrupo $T(t)=e^{A t}$ asociado al sistema (1)-(4) es exponencialmente estable, es decir, existen constantes positivas $M$ y $\delta$ tal que

$$
\left\|T(t) U_{0}\right\|_{\mathcal{H}} \leq M\left\|U_{0}\right\|_{\mathcal{H}} e^{-\delta t}, \forall t>0 .
$$

Demostración. Sea $U=(u, v, w, \theta)^{T} \in D(A)$ y $F=\left(f_{1}, f_{2}, f_{3}, f_{4}\right)^{T} \in \mathcal{H}$, de la ecuación resolvente

$$
(i \lambda U-A U)=F
$$

Se obtiene el siguiente sistema de ecuaciones

$$
\begin{aligned}
i \lambda u-v & =f_{1} \\
i \lambda v-w & =f_{2} \\
i \alpha \lambda w-\Delta a^{2} u-\Delta a^{2} \beta v+\Delta \eta \theta+w & =\alpha f_{3} \\
i \lambda \theta-\Delta \eta v-\Delta \alpha \eta w-\Delta \theta & =f_{4}
\end{aligned}
$$

Para demostrar el decaimiento exponencial del sistema, verificaremos la primera condición del teorema de Prüss; esto es $i \mathbb{R} \subset \rho(A)$. En efecto, desde que $A$ es un operador cerrado, $D(A)$ tiene inmersión compacta sobre el Espacio Fase $\mathcal{H}$ y $0 \in \rho(A)$ se tiene que $(\lambda I-A)^{-1}$ es compacto para todo $\lambda \in \rho(A)$. En particular, se tiene que $A^{-1}$ es compacto, puesto que $0 \in \rho(A)$. Por tanto, $\sigma\left(A^{-1}\right)$ esta conformado por autovalores y en consecuencia el espectro $\sigma(A)$ solo contiene autovalores.

Teniendo en cuenta el Teorema 6.29, ver Kato [8], para probar que $i \mathbb{R} \subset \rho(A)$ es suficiente mostrar que $\sigma(A)$ no contiene ningún autovalor imaginario. Procedemos por contradicción. Supongamos $i \mathbb{R} \not \subset \rho(A)$, es decir, que existe un autovalor imaginario $i \lambda, \lambda \in \mathbb{R}$, con $\lambda \neq 0$, entonces existe un autovector no nulo $U=(u, v, w, \theta) \in D(A)$ tal que

$$
A U=i \lambda U
$$

Tomando producto interno en la ecuación (20), obtenemos

$$
\langle A U, U\rangle_{\mathcal{H}}=-i \lambda\|U\|_{\mathcal{H}}^{2}
$$

Desde que el operador $A$ es disipativo y $(\beta-\alpha)>0$ se tiene

$$
\langle A U, U\rangle_{\mathcal{H}}=-a^{2}(\beta-\alpha) \int_{\Omega}\left|\nabla u_{t}\right|^{2} d x-\int_{\Omega}|\nabla \theta|^{2} d x \leq 0
$$


sustituyendo en (21), y tomando la parte real se concluye

$$
a^{2}(\beta-\alpha) \int_{\Omega}\left|\nabla u_{t}\right|^{2} d x+\int_{\Omega}|\nabla \theta|^{2} d x=\operatorname{Re}\langle A U, U\rangle_{\mathcal{H}}=0
$$

de donde

$$
a^{2}(\beta-\alpha) \int_{\Omega}\left|\nabla u_{t}\right|^{2} d x+\int_{\Omega}|\nabla \theta|^{2} d x=0
$$

luego $v=0$ y $\theta=0$.

A partir de la ecuación resolvente $(15)$ con $F=0$, obtenemos el siguiente sistema de ecuaciones

$$
\begin{aligned}
i \lambda u-v & =0 \\
i \lambda v-w & =0 \\
i \lambda \alpha w-a^{2} \Delta u-a^{2} \beta \Delta v+\eta \Delta \theta+w & =0 \\
i \lambda \theta-\eta \Delta v-\alpha \eta \Delta w-\Delta \theta & =0
\end{aligned}
$$

De 24) y 25) obtenemos $w=0$ y $u=0$, esto implica que $U=(u, v, w, \theta)^{T}=(0,0,0,0)^{T}$, lo que es una contradicción. Por lo tanto $i \mathbb{R} \subset \rho(A)$.

En lo que sigue demostraremos la segunda condición de teorema de Prüss, para ello efectuaremos estimativas para cada término de $\|U\|_{\mathcal{H}}^{2}$, donde

$$
\|U\|_{\mathcal{H}}^{2}=a^{2} \alpha(\beta-\alpha) \int_{\Omega}|\nabla v|^{2} d x+a^{2} \int_{\Omega}|\nabla u+\alpha \nabla v|^{2} d x+\int_{\Omega}|v+\alpha w|^{2} d x+\int_{\Omega}|\theta|^{2} d x
$$

Efectuando producto interno en (15) se obtiene

$$
i \lambda\|U\|_{\mathcal{H}}^{2}-\langle A U, U\rangle_{\mathcal{H}}=\langle F, U\rangle_{\mathcal{H}}
$$

luego, tomando la parte real, obtenemos

$$
-\operatorname{Re}\langle A U, U\rangle_{\mathcal{H}}=\operatorname{Re}\langle F, U\rangle_{\mathcal{H}}
$$

De (28) y (22), se obtiene

$$
a^{2} \alpha(\beta-\alpha) \int_{\Omega}|\nabla v|^{2} d x \leq C\|U\|_{\mathcal{H}}\|F\|_{\mathcal{H}}
$$

$\mathrm{y}$

$$
\int_{\Omega}|\nabla \theta|^{2} d x \leq C\|U\|_{\mathcal{H}}\|F\|_{\mathcal{H}}
$$

A partir de (30), por la desigualdad Poincaré, obtenemos

$$
\int_{\Omega}|\theta|^{2} d x \leq C\|U\|_{\mathcal{H}}\|F\|_{\mathcal{H}}
$$

En la ecuación 19 multiplicando por $\overline{v+\alpha w}$, integrando sobre $\Omega$, y aplicando el Teorema de Green se obtiene

$$
\eta \int_{\Omega}|\nabla(v+\alpha w)|^{2} d x=\int_{\Omega} f_{4}(\overline{v+\alpha w}) d x-\int_{\Omega} \nabla \theta . \nabla(\overline{v+\alpha w}) d x-i \lambda \int_{\Omega} \theta(\overline{v+\alpha w}) d x
$$

En seguida, tomando la parte real y aplicando la desigualdad triangular se tiene

$$
\begin{aligned}
\eta \int_{\Omega}|\nabla(v+\alpha w)|^{2} d x & \leq\left|\operatorname{Re}\left\{\int_{\Omega} f_{4}(\overline{v+\alpha w}) d x\right\}\right|+\left|\operatorname{Re}\left\{\int_{\Omega} \nabla \theta \cdot \nabla(\overline{v+\alpha w}) d x\right\}\right| \\
& +\left|\operatorname{Re}\left\{\int_{\Omega}(i \lambda \theta)(\overline{v+\alpha w}) d x\right\}\right|
\end{aligned}
$$


Aplicando la desigualdad de Young, Hölder, Poincaré y la equivalencia de normas en (32), se obtiene

$$
\begin{gathered}
\left|\operatorname{Re}\left\{\int_{\Omega} f_{4}(\overline{v+\alpha w}) d x\right\}\right| \leq C_{1}\|U\|_{\mathcal{H}}\|F\|_{\mathcal{H}}, \\
\left|\operatorname{Re}\left\{\int_{\Omega} \nabla \theta \cdot \nabla(\overline{v+\alpha w}) d x\right\}\right| \leq \frac{C}{2 \eta}\|U\|_{\mathcal{H}}\|F\|_{\mathcal{H}}+\frac{\eta}{2} \int_{\Omega}|\nabla(v+\alpha w)|^{2} d x
\end{gathered}
$$

$\mathrm{y}$

$$
\left|\operatorname{Re}\left\{\int_{\Omega}(i \lambda \theta)(\overline{v+\alpha w}) d x\right\}\right| \leq \frac{\lambda^{2} C}{\eta}\|U\|_{\mathcal{H}}\|F\|_{\mathcal{H}}+\frac{\eta}{4} \int_{\Omega}|\nabla(v+\alpha w)|^{2} d x
$$

Teniendo en cuenta (33)-(35), a partir de 32 se obtiene

$$
\frac{\eta}{4} \int_{\Omega}|\nabla(v+\alpha w)|^{2} d x \leq\left(C+\frac{C}{2 \eta}+\frac{\lambda^{2} C}{\eta}\right)\|U\|_{\mathcal{H}}\|F\|_{\mathcal{H}}
$$

De (36), por la desigualdad de Poincaré, se tiene

$$
\int_{\Omega}|v+\alpha w|^{2} d x \leq C\|U\|_{\mathcal{H}}\|F\|_{\mathcal{H}}
$$

Sustituyendo (16) y (17) en (18) se tiene

$$
i \alpha \lambda w-a^{2} \Delta u-i \lambda a^{2} \beta \Delta u+a^{2} \beta \Delta f_{1}+\eta \Delta \theta+i \lambda v-f_{2}=\alpha f_{3}
$$

Multiplicando por $\bar{u}$, e integramos sobre $\Omega$, obtenemos

$$
\begin{aligned}
i \alpha \lambda \int_{\Omega} w \bar{u} d x-a^{2} \int_{\Omega} \Delta u \bar{u} d x-i \lambda a^{2} \beta \int_{\Omega} \Delta u \bar{u} d x+a^{2} \beta \int_{\Omega} \Delta f_{1} \bar{u} d x \\
+\eta \int_{\Omega} \Delta \theta \bar{u} d x+i \lambda \int_{\Omega} v \bar{u} d x-\int_{\Omega} f_{2} \bar{u} d x=\alpha \int_{\Omega} f_{3} \bar{u} d x
\end{aligned}
$$

Aplicando nuevamente el Teorema de Green, se obtiene

$$
\begin{aligned}
a^{2} \int_{\Omega}|\nabla u|^{2} d x & \leq a^{2} \beta \int_{\Omega} \nabla f_{1} \cdot \nabla \bar{u} d x+\eta \int_{\Omega} \nabla \theta \cdot \nabla \bar{u} d x+\int_{\Omega} f_{2} \bar{u} d x \\
& +\alpha \int_{\Omega} f_{3} \bar{u} d x-i \lambda \int_{\Omega}(v+\alpha w) \bar{u} d x-i \lambda a^{2} \beta \int_{\Omega}|\nabla u|^{2} d x
\end{aligned}
$$

De modo similar que en 32 , efectuando estimaciones, obtenemos

$$
\int_{\Omega}|\nabla u|^{2} d x \leq \widetilde{C}\|U\|_{\mathcal{H}}\|F\|_{\mathcal{H}}
$$

Por último, teniendo en cuenta (29) y (38) se obtiene

$$
\int_{\Omega}|\nabla u+\alpha \nabla v|^{2} d x \leq \int_{\Omega}|\nabla u|^{2} d x+\alpha^{2} \int_{\Omega}|\nabla v|^{2} d x \leq\left(\widetilde{C}+\frac{C}{(\beta-\alpha)}\right)\|U\|_{\mathcal{H}}\|F\|_{\mathcal{H}}
$$

de donde se tiene

$$
a^{2} \int_{\Omega}|\nabla u+\alpha \nabla v|^{2} d x \leq C\|U\|_{\mathcal{H}}\|F\|_{\mathcal{H}}
$$

Sumando (29), (31), 37) y (39) se obtiene

$$
\|U\|_{\mathcal{H}}^{2}=C\|U\|_{\mathcal{H}}\|F\|_{\mathcal{H}} \text { con } C>0
$$

De donde se conluye

$$
\|U\|_{\mathcal{H}} \leq C\|F\|_{\mathcal{H}}
$$

Por tanto

$$
\left\|(i \lambda I-A)^{-1}\right\|_{L(\mathcal{H})} \leq C, \forall|\lambda|>1
$$




\section{Discretización de la energía}

En esta sección vamos a verificar el decaimiento exponencial del sistema (11)-(4) para el caso unidimensional, para determinar la energía discretizada haremos uso del método de diferencias finitas. Teniendo en cuenta los trabajos de Santos et al. [15], Negreanu et al. 9] y Schiavi et al. [16].

Realizamos la discretización del sistema (1)-(4) por el método de diferencias finitas para el caso unidimensional, tenemos el siguiente sistema:

$$
\begin{aligned}
\alpha u_{t t t}+u_{t t}-a^{2} u_{x x}-a^{2} \beta u_{x x t}+\eta \theta_{x x} & =0, \text { en }] 0, L[\times] 0, \infty[ \\
\theta_{t}-\theta_{x x}-\alpha \eta u_{x x t t}-\eta u_{x x t} & =0, \text { en }] 0, L[\times] 0, \infty[
\end{aligned}
$$

Con condiciones iniciales

$$
\begin{aligned}
u(x, 0) & \left.=u_{0}(x), u_{t}(x, 0)=u_{1}(x), \forall x \in\right] 0, L[ \\
u_{t t}(x, 0) & \left.=u_{2}(x), \theta(x, 0)=\theta_{0}(x), \forall x \in\right] 0, L[
\end{aligned}
$$

y condiciones de frontera

$$
\left.u(0, t)=u(L, t)=u_{t}(0, t)=u_{t}(L, t)=\theta(0, t)=\theta(L, t)=0, t \in\right] 0, T[
$$

Hacemos el cambio $\varphi=\alpha u_{t}+u$, y reemplazamos en (40) y (41), obtenemos

$$
\begin{aligned}
& \varphi_{t t}-a^{2} \varphi_{x x}+a^{2}(\alpha-\beta) u_{x x t}+\eta \theta_{x x}=0 \\
& \theta_{t}-\theta_{x x}-\eta \varphi_{x x t}=0
\end{aligned}
$$

Con condiciones iniciales

$$
\begin{aligned}
u(x, 0) & \left.=u_{0}(x), u_{t}(x, 0)=u_{1}(x), \forall x \in\right] 0, L[ \\
u_{t t}(x, 0) & \left.=u_{2}(x), \theta(x, 0)=\theta_{0}(x), \forall x \in\right] 0, L[ \\
\varphi(x, 0) & \left.=\alpha u_{t}(x, 0)+u(x, 0)=\varphi_{0}(x), \forall x \in\right] 0, L[ \\
\varphi_{t}(x, 0) & \left.=\alpha u_{t t}(x, 0)+u_{t}(x, 0)=\varphi_{1}(x), \forall x \in\right] 0, L[
\end{aligned}
$$

Con condiciones de frontera

$$
\begin{aligned}
& \left.u(0, t)=u(L, t)=u_{t}(0, t)=u_{t}(L, t)=\theta(0, t)=\theta(L, t)=0, t \in\right] 0, T[ \\
& \left.\varphi(0, t)=\alpha u_{t}(0, t)+u(0, t)=0, \quad \varphi(L, t)=\alpha u_{t}(L, t)+u(L, t)=0, \quad t \in\right] 0, T[
\end{aligned}
$$

\subsection{Método numérico en diferencias finitas}

El sistema (45)-(52) lo discretizaremos en la variable espacial $x$ y temporal $t$ por el método de diferencias finitas. Definimos la siguiente malla.

$$
\begin{aligned}
& x_{0}=0<x_{1}=\Delta x<\ldots<x_{J}=J \Delta x<x_{J+1}=L \\
& t_{0}=0<t_{1}=\Delta t<\ldots<t_{N}=N \Delta t<t_{N+1}=T
\end{aligned}
$$

Donde

$$
\Delta x=\frac{L}{J+1} \quad \text { y } \quad \Delta t=\frac{T}{N+1}, \quad \text { para } \quad J, N \in N
$$

Denotaremos por $u_{j}^{n}, \varphi_{j}^{n}$ y $\theta_{j}^{n}$ las soluciones numéricas en los puntos nodales $\left(x_{j}, t_{n}\right)$ de la discretización, sus aproximaciones numéricas para las soluciones exactas $u, \varphi$ y $\theta$ que trabajamos en la malla. De modo más precisa tenemos que

$$
u_{j}^{n} \approx u\left(x_{j}, t_{n}\right), \quad \varphi_{j}^{n} \approx \varphi\left(x_{j}, t_{n}\right) \quad \text { y } \quad \theta_{j}^{n} \approx \theta\left(x_{j}, t_{n}\right)
$$


Proposición 2 La fórmula en diferencias finitas de $u_{x x t}(x, t)$ está dada por

$$
u_{x x t}\left(x_{j}, t_{n}\right) \approx \frac{u_{j+1}^{n+1}-2 u_{j}^{n+1}+u_{j-1}^{n+1}}{2 \Delta t \Delta x^{2}}-\frac{u_{j+1}^{n-1}-2 u_{j}^{n-1}+u_{j-1}^{n-1}}{2 \Delta t \Delta x^{2}}
$$

con un error de orden $O\left(\frac{(\Delta x)^{2}}{\Delta t}, \Delta x, \Delta t, \frac{(\Delta t)^{2}}{\Delta x}, \frac{(\Delta t)^{3}}{(\Delta x)^{2}}, \frac{(\Delta t)^{4}}{(\Delta x)^{2}}\right)$

Demostración. Teniendo en cuenta el desarrollo de las series de Taylor en dos variables y siguiendo como referencia el trabajo de Schiavi et al.[16], se puede deselvolver la proposición, debido a que es extenso omitimos los detalles del resultado, para el lector que quiera investigar en [14].

Consideremos los siguientes operadores de diferencias finitas en espacio y en el tiempo:

$$
\begin{aligned}
& \bar{\partial}_{t} \partial_{t} u_{j}^{n}=\frac{u_{j}^{n+1}-2 u_{j}^{n}+u_{j}^{n-1}}{\Delta t^{2}}, \quad \bar{\partial}_{x} \partial_{x} u_{j}^{n}=\frac{u_{j+1}^{n}-2 u_{j}^{n}+u_{j-1}^{n}}{\Delta x^{2}} \\
& \varphi_{j}^{n}=\alpha\left(\frac{u_{j}^{n+1}-u_{j}^{n-1}}{2 \Delta t}\right)+u_{j}^{n} \quad \bar{\partial}_{t} u_{j}^{n}=\frac{u_{j}^{n}-u_{j}^{n-1}}{\Delta t}, \\
& \frac{\partial^{3}}{\partial x^{2} \partial t} u_{j}^{n}=\frac{u_{j+1}^{n+1}-2 u_{j}^{n+1}+u_{j-1}^{n+1}}{2 \Delta t \Delta x^{2}}-\frac{u_{j+1}^{n-1}-2 u_{j}^{n-1}+u_{j-1}^{n-1}}{2 \Delta t \Delta x^{2}}
\end{aligned}
$$

Considerando la discretización de $\theta$ como

$$
\begin{aligned}
& \theta\left(x_{j}, t_{n}\right) \approx \frac{\theta\left(x_{j}, t_{n}-\Delta t\right)+\theta\left(x_{j}, t_{n}\right)}{2}+O(\Delta t) \\
& \theta\left(x_{j}, t_{n}\right) \approx \frac{\theta_{j}^{n-1}+\theta_{j}^{n}}{2} \text { con error } O(\Delta t)
\end{aligned}
$$

El esquema numérico espacio - tiempo en diferencias finitas del sistema 45- 46 está dado por las siguientes ecuaciones numéricas.

$$
\begin{aligned}
\bar{\partial}_{t} \partial_{t} \varphi_{j}^{n}-a^{2} \bar{\partial}_{x} \partial_{x} \varphi_{j}^{n}+a^{2}(\alpha-\beta) \frac{\partial^{3}}{\partial x^{2} \partial t} u_{j}^{n}+\eta \bar{\partial}_{x} \partial_{x} \theta_{j}^{n}=0 \\
\bar{\partial}_{t} \theta_{j}^{n}-\bar{\partial}_{x} \partial_{x} \theta_{j}^{n}-\eta \frac{\partial^{3}}{\partial x^{2} \partial t} \varphi_{j}^{n}=0
\end{aligned}
$$

Teniendo en cuenta los siguientes operadores discretizados de (55)-(58) se obtiene el siguiente esquema numérico

$$
\begin{gathered}
\frac{\varphi_{j}^{n+1}-2 \varphi_{j}^{n}+\varphi_{j}^{n-1}}{\Delta t^{2}}-a^{2}\left(\frac{\varphi_{j+1}^{n}-2 \varphi_{j}^{n}+\varphi_{j-1}^{n}}{\Delta x^{2}}\right)+\eta\left(\frac{\theta_{j+1}^{n}-2 \theta_{j}^{n}+\theta_{j-1}^{n}}{\Delta x^{2}}\right) \\
+a^{2}(\alpha-\beta)\left(\frac{u_{j+1}^{n+1}-2 u_{j}^{n+1}+u_{j-1}^{n+1}}{2 \Delta t \Delta x^{2}}-\frac{u_{j+1}^{n-1}-2 u_{j}^{n-1}+u_{j-1}^{n-1}}{2 \Delta t \Delta x^{2}}\right)=0 \\
\frac{\theta_{j}^{n}-\theta_{j}^{n-1}}{\Delta t}-\frac{\theta_{j+1}^{n}-2 \theta_{j}^{n}+\theta_{j-1}^{n}}{\Delta x^{2}}-\eta\left(\frac{\varphi_{j+1}^{n+1}-2 \varphi_{j}^{n+1}+\varphi_{j-1}^{n+1}}{2 \Delta t \Delta x^{2}}-\frac{\varphi_{j+1}^{n-1}-2 \varphi_{j}^{n-1}+\varphi_{j-1}^{n-1}}{2 \Delta t \Delta x^{2}}\right)=0
\end{gathered}
$$

Para todo $j=1,2, \ldots, J$ y $n=1,2, \ldots, N$. Con las condiciones de frontera dadas por

$$
u_{0}^{n}=u_{J+1}^{n}=0, \quad \theta_{0}^{n}=\theta_{J+1}^{n}=0, \quad \varphi_{0}^{n}=\varphi_{J+1}^{n}=0, \quad \forall n=1,2, \ldots, N
$$


La discretización de las condiciones iniciales están dadas por

$$
\begin{array}{lll}
u_{j}^{0}=u\left(x_{j}, 0\right), & u_{j}^{1}=u_{j}^{-1}+2 \Delta t u_{t}\left(x_{j}, 0\right), & \forall j=1,2, \ldots, J \\
\varphi_{j}^{0}=\varphi\left(x_{j}, 0\right), & \varphi_{j}^{1}=\varphi_{j}^{-1}+2 \Delta t \varphi_{t}\left(x_{j}, 0\right), & \forall j=1,2, \ldots, J \\
\theta_{j}^{0}=\theta\left(x_{j}, 0\right), & \forall j=1,2, \ldots, J &
\end{array}
$$

La energía numérica del sistema (61)-(66) está definida por

$$
\begin{aligned}
E^{n} & =\frac{\Delta x}{2} \sum_{j=0}^{J}\left[\left\{\alpha\left(\frac{u_{j}^{n+2}-u_{j}^{n}}{2 \Delta t^{2}}-\frac{u_{j}^{n+1}-u_{j}^{n-1}}{2 \Delta t^{2}}\right)+\frac{u_{j}^{n+1}-u_{j}^{n}}{\Delta t}\right\}^{2}\right. \\
& +a^{2}\left[\alpha\left(\frac{u_{j+1}^{n+2}-u_{j+1}^{n}}{2 \Delta t \Delta x}-\frac{u_{j}^{n+2}-u_{j}^{n}}{2 \Delta t \Delta x}\right)+\frac{u_{j+1}^{n+1}-u_{j}^{n+1}}{\Delta x}\right] . \\
& {\left[\alpha\left(\frac{u_{j+1}^{n+1}-u_{j+1}^{n-1}}{2 \Delta t \Delta x}-\frac{u_{j}^{n+1}-u_{j}^{n-1}}{2 \Delta t \Delta x}\right)+\frac{u_{j+1}^{n}-u_{j}^{n}}{\Delta x}\right]+\left(\theta_{j}^{n}\right)^{2} } \\
& \left.+a^{2}(\beta-\alpha) \alpha\left[\frac{\left(u_{j+1}^{n+2}-u_{j+1}^{n}\right)-\left(u_{j}^{n+2}-u_{j}^{n}\right)}{2 \Delta t \Delta x}\right]\left[\frac{\left(u_{j+1}^{n+1}-u_{j+1}^{n-1}\right)-\left(u_{j}^{n+1}-u_{j}^{n-1}\right)}{2 \Delta t \Delta x}\right]\right]
\end{aligned}
$$

Para todo $n=1,2, \ldots, N$

Proposición 3 Sea $\left(u_{j}^{n}, \theta_{j}^{n}\right)$ la solución del sistema discreto 61 - 66 la tasa discreta de variación de la energía en el instante $t_{n}$ es dada por

$$
\frac{E^{n}-E^{n-1}}{\Delta t}=-\sum_{j=0}^{J}\left(\frac{\theta_{j+1}^{n}-\theta_{j}^{n}}{\Delta x}\right)^{2}-\frac{a^{2}(\beta-\alpha)}{4 \Delta t^{2} \Delta x^{2}} \sum_{j=0}^{J}\left[\left(u_{j+1}^{n+1}-u_{j+1}^{n-1}\right)-\left(u_{j}^{n+1}-u_{j}^{n-1}\right)\right]^{2}
$$

$\forall n \in 1,2, \ldots, N$.

Demostración. Multiplicando las ecuaciones 61$), 62$ por $\frac{1}{2}\left(\varphi_{j}^{n+1}-\varphi_{j}^{n-1}\right)$ y $\Delta t \theta_{j}^{n}$, respectivamente, luego sumando en el dominio discreto, para $j=1,2, \ldots, J$, se obtiene

$$
\begin{gathered}
\frac{1}{2} \sum_{j=1}^{J}\left(\frac{\varphi_{j}^{n+1}-2 \varphi_{j}^{n}+\varphi_{j}^{n-1}}{\Delta t^{2}}\right)\left(\varphi_{j}^{n+1}-\varphi_{j}^{n-1}\right)-\frac{a^{2}}{2} \sum_{j=1}^{J}\left(\frac{\varphi_{j+1}^{n}-2 \varphi_{j}^{n}+\varphi_{j-1}^{n}}{\Delta x^{2}}\right)\left(\varphi_{j}^{n+1}-\varphi_{j}^{n-1}\right) \\
+\frac{a^{2}(\alpha-\beta)}{2} \sum_{j=1}^{J}\left(\frac{u_{j+1}^{n+1}-2 u_{j}^{n+1}+u_{j-1}^{n+1}}{2 \Delta t \Delta x^{2}}-\frac{u_{j+1}^{n-1}-2 u_{j}^{n-1}+u_{j-1}^{n-1}}{2 \Delta t \Delta x^{2}}\right)\left(\varphi_{j}^{n+1}-\varphi_{j}^{n-1}\right) \\
+\frac{\eta}{2} \sum_{j=1}^{J}\left(\frac{\theta_{j+1}^{n}-2 \theta_{j}^{n}+\theta_{j-1}^{n}}{\Delta x^{2}}\right)\left(\varphi_{j}^{n+1}-\varphi_{j}^{n-1}\right)=0
\end{gathered}
$$

$\mathrm{y}$

$$
\begin{aligned}
\sum_{j=1}^{J}\left(\frac{\theta_{j}^{n}-\theta_{j}^{n-1}}{\Delta t}\right) & \Delta t \theta_{j}^{n}-\sum_{j=1}^{J}\left(\frac{\theta_{j+1}^{n}-2 \theta_{j}^{n}+\theta_{j-1}^{n}}{\Delta x^{2}}\right) \Delta t \theta_{j}^{n} \\
& -\eta \sum_{j=1}^{J}\left(\frac{\varphi_{j+1}^{n+1}-2 \varphi_{j}^{n+1}+\varphi_{j-1}^{n+1}}{2 \Delta t \Delta x^{2}}-\frac{\varphi_{j+1}^{n-1}-2 \varphi_{j}^{n-1}+\varphi_{j-1}^{n-1}}{2 \Delta t \Delta x^{2}}\right) \Delta t \theta_{j}^{n}=0
\end{aligned}
$$


Considerando como referencia el trabajo de Negreanu et al. [9], de (69)-(70) considerando las condiciones de frontera discreta se obtienen los siguientes resultados

$$
\begin{aligned}
\frac{1}{2} \sum_{j=1}^{J}\left(\frac{\varphi_{j}^{n+1}-2 \varphi_{j}^{n}+\varphi_{j}^{n-1}}{\Delta t^{2}}\right)\left(\varphi_{j}^{n+1}-\varphi_{j}^{n-1}\right)=\frac{1}{2} \sum_{j=0}^{J}\left(\frac{\varphi_{j}^{n+1}-\varphi_{j}^{n}}{\Delta t}\right)^{2} \\
-\frac{1}{2} \sum_{j=0}^{J}\left(\frac{\varphi_{j}^{n}-\varphi_{j}^{n-1}}{\Delta t}\right)^{2},
\end{aligned}
$$

$$
\begin{aligned}
-\frac{a^{2}}{2} \sum_{j=1}^{J}\left(\frac{\varphi_{j+1}^{n}-2 \varphi_{j}^{n}+\varphi_{j-1}^{n}}{\Delta x^{2}}\right)\left(\varphi_{j}^{n+1}-\varphi_{j}^{n-1}\right) & =\frac{a^{2}}{2} \sum_{j=0}^{J}\left(\frac{\varphi_{j+1}^{n+1}-\varphi_{j}^{n+1}}{\Delta x}\right)\left(\frac{\varphi_{j+1}^{n}-\varphi_{j}^{n}}{\Delta x}\right) \\
& -\frac{a^{2}}{2} \sum_{j=0}^{J}\left(\frac{\varphi_{j+1}^{n}-\varphi_{j}^{n}}{\Delta x}\right)\left(\frac{\varphi_{j+1}^{n-1}-\varphi_{j}^{n-1}}{\Delta x}\right),
\end{aligned}
$$

$$
\begin{aligned}
-\eta \sum_{j=1}^{J}\left(\frac{\varphi_{j+1}^{n+1}-2 \varphi_{j}^{n+1}+\varphi_{j-1}^{n+1}}{2 \Delta t \Delta x^{2}}-\right. & \left.\frac{\varphi_{j+1}^{n-1}-2 \varphi_{j}^{n-1}+\varphi_{j-1}^{n-1}}{2 \Delta t \Delta x^{2}}\right) \Delta t \theta_{j}^{n} \\
& +\frac{\eta}{2} \sum_{j=1}^{J}\left(\frac{\theta_{j+1}^{n}-2 \theta_{j}^{n}+\theta_{j-1}^{n}}{\Delta x^{2}}\right)\left(\varphi_{j}^{n+1}-\varphi_{j}^{n-1}\right)=0,
\end{aligned}
$$

$$
\begin{gathered}
\sum_{j=1}^{J}\left(\frac{\theta_{j}^{n}-\theta_{j}^{n-1}}{\Delta t}\right) \Delta t \theta_{j}^{n}=\frac{1}{2} \sum_{j=0}^{J}\left(\theta_{j}^{n}\right)^{2}-\frac{1}{2} \sum_{j=0}^{J}\left(\theta_{j}^{n-1}\right)^{2}, \\
-\sum_{j=1}^{J}\left(\frac{\theta_{j+1}^{n}-2 \theta_{j}^{n}+\theta_{j-1}^{n}}{\Delta x^{2}}\right) \Delta t \theta_{j}^{n}=\Delta t \sum_{j=0}^{J}\left(\frac{\theta_{j+1}^{n}-\theta_{j}^{n}}{\Delta x}\right)^{2},
\end{gathered}
$$

$\mathrm{y}$

$$
\begin{aligned}
& \frac{a^{2}(\alpha-\beta)}{2} \sum_{j=1}^{J}\left(\frac{u_{j+1}^{n+1}-2 u_{j}^{n+1}+u_{j-1}^{n+1}}{2 \Delta t \Delta x^{2}}-\frac{u_{j+1}^{n-1}-2 u_{j}^{n-1}+u_{j-1}^{n-1}}{2 \Delta t \Delta x^{2}}\right)\left(\varphi_{j}^{n+1}-\varphi_{j}^{n-1}\right) \\
& =\frac{a^{2}(\beta-\alpha) \alpha}{2} \sum_{j=0}^{J}\left[\frac{\left(u_{j+1}^{n+2}-u_{j+1}^{n}\right)-\left(u_{j}^{n+2}-u_{j}^{n}\right)}{2 \Delta t \Delta x}\right]\left[\frac{\left(u_{j+1}^{n+1}-u_{j+1}^{n-1}\right)-\left(u_{j}^{n+1}-u_{j}^{n-1}\right)}{2 \Delta t \Delta x}\right] \\
& -\frac{a^{2}(\beta-\alpha) \alpha}{2} \sum_{j=0}^{J}\left[\frac{\left(u_{j+1}^{n+1}-u_{j+1}^{n-1}\right)-\left(u_{j}^{n+1}-u_{j}^{n-1}\right)}{2 \Delta t \Delta x}\right]\left[\frac{\left(u_{j+1}^{n}-u_{j+1}^{n-2}\right)-\left(u_{j}^{n}-u_{j}^{n-2}\right)}{2 \Delta t \Delta x}\right] \\
& +\frac{a^{2}(\beta-\alpha)}{4 \Delta t \Delta x^{2}} \sum_{j=0}^{J}\left[\left(u_{j+1}^{n+1}-u_{j+1}^{n-1}\right)-\left(u_{j}^{n+1}-u_{j}^{n-1}\right)\right]^{2},
\end{aligned}
$$


Sumando las expresiones (71) a (76) obtenemos la siguiente expresión

$$
\begin{aligned}
& \frac{1}{2} \sum_{j=0}^{J}\left(\frac{\varphi_{j}^{n+1}-\varphi_{j}^{n}}{\Delta t}\right)^{2}+\frac{1}{2} \sum_{j=0}^{J}\left(\theta_{j}^{n}\right)^{2}+\frac{a^{2}}{2} \sum_{j=0}^{J}\left(\frac{\varphi_{j+1}^{n+1}-\varphi_{j}^{n+1}}{\Delta x}\right)\left(\frac{\varphi_{j+1}^{n}-\varphi_{j}^{n}}{\Delta x}\right) \\
& +\frac{a^{2}(\beta-\alpha) \alpha}{2} \sum_{j=0}^{J}\left[\frac{\left(u_{j+1}^{n+2}-u_{j+1}^{n}\right)-\left(u_{j}^{n+2}-u_{j}^{n}\right)}{2 \Delta t \Delta x}\right]\left[\frac{\left(u_{j+1}^{n+1}-u_{j+1}^{n-1}\right)-\left(u_{j}^{n+1}-u_{j}^{n-1}\right)}{2 \Delta t \Delta x}\right] \\
& -\left(\frac{1}{2} \sum_{j=0}^{J}\left(\frac{\varphi_{j}^{n}-\varphi_{j}^{n-1}}{\Delta t}\right)^{2}+\frac{1}{2} \sum_{j=0}^{J}\left(\theta_{j}^{n-1}\right)^{2}+\frac{a^{2}}{2} \sum_{j=0}^{J}\left(\frac{\varphi_{j+1}^{n}-\varphi_{j}^{n}}{\Delta x}\right)\left(\frac{\varphi_{j+1}^{n-1}-\varphi_{j}^{n-1}}{\Delta x}\right)\right. \\
& \left.+\frac{a^{2}(\beta-\alpha) \alpha}{2} \sum_{j=0}^{J}\left[\frac{\left(u_{j+1}^{n+1}-u_{j+1}^{n-1}\right)-\left(u_{j}^{n+1}-u_{j}^{n-1}\right)}{2 \Delta t \Delta x}\right]\left[\frac{\left(u_{j+1}^{n}-u_{j+1}^{n-2}\right)-\left(u_{j}^{n}-u_{j}^{n-2}\right)}{2 \Delta t \Delta x}\right]\right) \\
& +\frac{a^{2}(\beta-\alpha)}{4 \Delta t \Delta x^{2}} \sum_{j=0}^{J}\left[\left(u_{j+1}^{n+1}-u_{j+1}^{n-1}\right)-\left(u_{j}^{n+1}-u_{j}^{n-1}\right)\right]^{2}+\Delta t \sum_{j=0}^{J}\left(\frac{\theta_{j+1}^{n}-\theta_{j}^{n}}{\Delta x}\right)^{2}=0
\end{aligned}
$$

De la definición de (67) se tiene

$$
E^{n}+\Delta t \sum_{j=0}^{J}\left(\frac{\theta_{j+1}^{n}-\theta_{j}^{n}}{\Delta x}\right)^{2}+\frac{a^{2}(\beta-\alpha)}{4 \Delta t \Delta x^{2}} \sum_{j=0}^{J}\left[\left(u_{j+1}^{n+1}-u_{j+1}^{n-1}\right)-\left(u_{j}^{n+1}-u_{j}^{n-1}\right)\right]^{2}=E^{n-1}
$$

de donde se demuestra

$$
\frac{E^{n}-E^{n-1}}{\Delta t}=-\sum_{j=0}^{J}\left(\frac{\theta_{j+1}^{n}-\theta_{j}^{n}}{\Delta x}\right)^{2}-\frac{a^{2}(\beta-\alpha)}{4 \Delta t^{2} \Delta x^{2}} \sum_{j=0}^{J}\left[\left(u_{j+1}^{n+1}-u_{j+1}^{n-1}\right)-\left(u_{j}^{n+1}-u_{j}^{n-1}\right)\right]^{2}
$$

$\forall n \in 1,2, \ldots, N$.

Lo que completa la demostración de la proposición.

Proposición 4 La energía de las soluciones de las ecuaciones discretas 61)- 62 con las condiciones de contorno, se cumple que

$$
E^{n} \leq E^{0}, \quad \forall n=1,2, \ldots, N .
$$

Demostración. De (78), obtenemos

$$
E^{n} \leq E^{n-1}
$$

Haciendo un procedimiento recursivo es fácil de obtener

$$
E^{n} \leq E^{0}, \quad \forall n=1,2, \ldots, N .
$$

Con la cual se concluye la demostración.

Ahora vamos a despejar $u_{j}^{n+1}, \varphi_{j}^{n+1}$ y $\theta_{j}^{n}$. De la expresión 56 despejando $u_{j}^{n+1}$, se obtine

$$
u_{j}^{n+1}=\frac{2 \Delta t}{\alpha} \varphi_{j}^{n}+u_{j}^{n-1}-\frac{2 \Delta t}{\alpha} u_{j}^{n}
$$

De la expresión 61 despejando $\varphi_{j}^{n+1}$, obtenemos

$$
\begin{aligned}
\varphi_{j}^{n+1}= & \left(b_{1} \varphi_{j-1}^{n}+a_{1} \varphi_{j}^{n}+b_{1} \varphi_{j+1}^{n}\right)-\varphi_{j}^{n-1}+c_{1}\left(u_{j-1}^{n+1}-2 u_{j}^{n+1}+u_{j+1}^{n+1}\right) \\
& -c_{1}\left(u_{j-1}^{n-1}-2 u_{j}^{n-1}+u_{j+1}^{n-1}\right)-d_{1}\left(\theta_{j-1}^{n}-2 \theta_{j}^{n}+\theta_{j+1}^{n}\right)
\end{aligned}
$$


De la expresión (62) se obtine

$$
-f_{1} \theta_{j-1}^{n}+e_{1} \theta_{j}^{n}-f_{1} \theta_{j+1}^{n}=\theta_{j}^{n-1}+g_{1}\left(\varphi_{j-1}^{n+1}-2 \varphi_{j}^{n+1}+\varphi_{j+1}^{n+1}\right)-g_{1}\left(\varphi_{j-1}^{n-1}-2 \varphi_{j}^{n-1}+\varphi_{j+1}^{n-1}\right)
$$

Para efectos de cálculos computacionales usamos los términos obtenidos en (79), 80, y 81) donde

$$
\begin{array}{rlrl}
a_{1}=2-\frac{2 a^{2} \Delta t^{2}}{\Delta x^{2}}, & b_{1}=\frac{a^{2} \Delta t^{2}}{\Delta x^{2}}, & c_{1}=\frac{a^{2}(\beta-\alpha) \Delta t}{2 \Delta x^{2}}, & d_{1}=\frac{\eta \Delta t^{2}}{\Delta x^{2}}, \\
e_{1}=1+\frac{2 \Delta t}{\Delta x^{2}}, & f_{1}=\frac{\Delta t}{\Delta x^{2}}, & g_{1}=\frac{\eta}{2 \Delta x^{2}},
\end{array}
$$

\subsection{Simulación numérica}

A continuación mostraremos la simulación numérica del sistema (61)- (66), así como de la energía $E^{n}$ dado en (67). Para las simulaciones numéricas hemos considerado los siguientes valores

$$
a=0,6, L=1, T=2,5, \beta=0,75, \alpha=0,35, \eta=0,60
$$

Considerando las condiciones iniciales

$$
\begin{aligned}
& u\left(x_{j}, 0\right)=0, \quad \theta\left(x_{j}, 0\right)=\operatorname{sen}\left(\frac{3 \pi x_{j}}{L}\right), \quad u_{t}\left(x_{j}, 0\right)=\operatorname{sen}\left(\frac{3 \pi x_{j}}{L}\right) \\
& u_{t t}\left(x_{j}, 0\right)=\operatorname{sen}\left(\frac{2 \pi x_{j}}{L}\right), \quad \varphi\left(x_{j}, 0\right)=\alpha u_{t}\left(x_{j}, 0\right)+u\left(x_{j}, 0\right)
\end{aligned}
$$

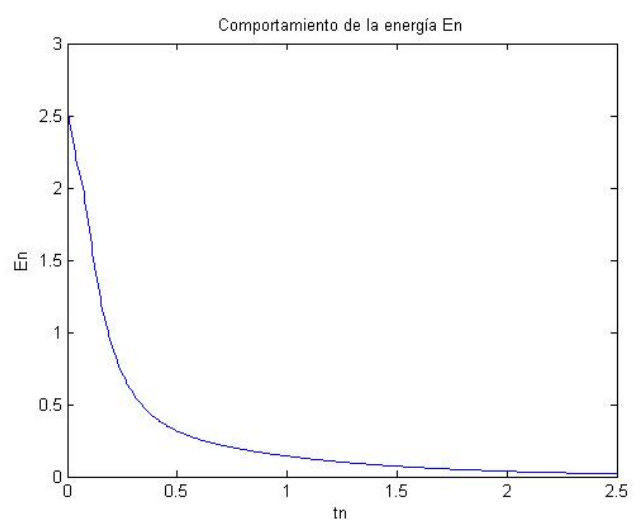

Figura 1: Decaimiento de la energía. $\alpha=0,35$

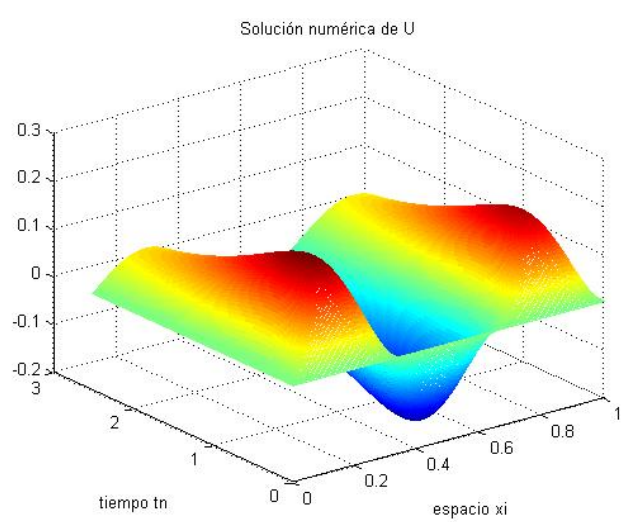

Figura 2: Vibración de la estructura 


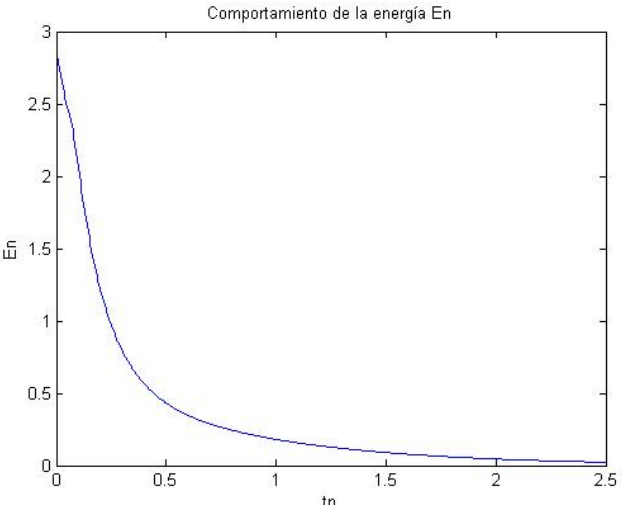

Figura 3: Decaimiento de la energía. $\alpha=0,40$

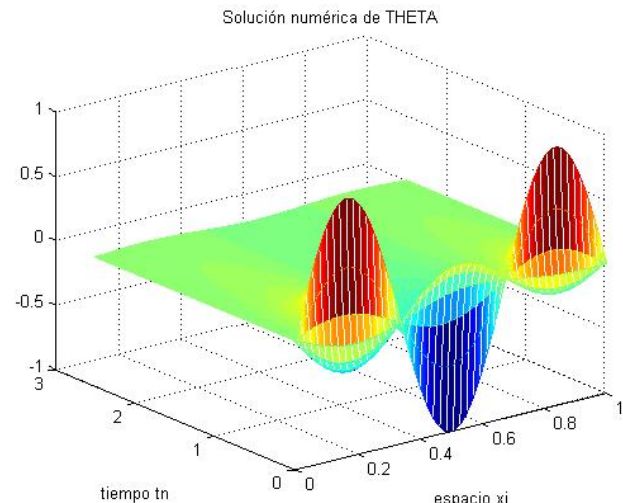

Figura 4: Diferencia de temperatura

En las figuras, observamos que la energía decae exponencialmente, de las figuras, podemos notar el comportamiento oscilatorio, que conforme transcurre el tiempo las soluciones se aproximan a cero.

\section{Conclusiones}

- Usando el método de Prüss se demostró que el sistema (1)-(4) decae exponencialmente también se puede estudiar el decaimiento exponencial con el método de la energía.

- Con el método de diferencias finitas se discretizo la energía del sistema para el caso unidimenssional y se comrpobo que la energía decrece conforme trascurre el tiempo.

- En lo que respecta a la implementación computacional observamos que la energía para el caso unidimensional decae exponencialmente.

- Se considero que $\Delta x=\Delta t$ para que se pueda controlar el error de discretización y sea del orden $O(\Delta t)$.

- Para estudios futuros se puede discretizar el mismo sistema pero en dos dimensiones y determinar la energía discretizada. 


\section{Referencias bibliográficas}

[1] Adams, R. (1975). Sobolev Spaces. United States of America, New York: Academic Press.

[2] Alves, M.S.; Buriol C.; Ferreira, M.V.; Muñoz, J.E.; Sepúlveda, M.; Vera O. (2013) Asymptotic behaviour for the vibrations modeled by the standard linear solid model with a termal effect, J. Math. Anal. Appl. 399 (2013) pp. 472-479.

[3] Bose, Sujit K.; Gorain, Ganesh C. Stability of the boundary stabilised internally damped wave equation in a bounded domain in $\mathbb{R}^{n}$, Block JD, Sector III, Salt Lake City, Calcutta (1998).

[4] Brezis, H. (1983). Analyse Fonctionnelle - Théorie et Applications. Francia, Paris: Mason.

[5] G. C. Gorain, Exponential energy decay estimate for the solutions of internally damped wave equation in a bounded domain, J. Math. Anal. Appl. 216 (1997) 510-520.

[6] Fatori, L.; Muñoz, J.E. Rates of decay to weak thermoelastic Bresse system, IMA J. Appl. Math., (2010), pp. 1-24.

[7] Fung, Y.C. Foundation of Solid Mechamics, Prentice-Hall, New Delhi (1968).

[8] Kato,T. Perturbation Theory for Linear Operators, Springer - Verlag, Berlin Heidelberg, (1980)

[9] Negreanu, M.; Zuazua, E. Uniform boundary controllability of a discrete 1-D wave equation. Preprint submitted to Elsevier Science, (Spain), (2002).

[10] Z. Liu and S. Zheng. Semigroups Associated with Dissipative Systems. In CRC Research Notes in Mathematics 398. Chapman and Hall. (1999).

[11] Pazy, A. Semigroups of linear operators and applications to partial differential equations, Springer-Verlag, New York, NY, (1983).

[12] Prüss, J. On the spectrum of-semigroups, Trans Amer. Math. Soc. 28, pp. 847-857, (1984).

[13] Rabotnov, Y.N. Elements of Hereditary Solid Mechamics, MIR Publication, Moscov, (1980).

[14] Romero, C. Comportamiento asintótico, discretización por diferencia finita y simulación numérica para el modelo de vibración de un sólido lineal estándar con efecto térmico, Repositorio Pesquimat

[15] Santos, M.L. and Almeida, D.S. Numerical exponential decay to dissipative Bresse system, J. Mth. Appl. Vol. 2010.

[16] Schiavi, E.; Muñoz, A.I. y Conde, C. Métodos en diferencias finitas para la resolución de problemas de contorno, (2006-2007) 